\title{
Anti-neuropathic pain activity of a cationic palladium (II) dithiocarbamate by suppressing the inflammatory mediators in paclitaxel-induced neuropathic pain model
}

\author{
Muhammad Naveed $^{1,2} \cdot$ Rahim Ullah ${ }^{3} \cdot$ Adnan Khan $^{2} \cdot$ Bushra Shal $^{2} \cdot$ Ashraf Ullah Khan $^{2} \cdot$ Shahan Zeb Khan ${ }^{4,5}$. \\ Zia ur Rehman ${ }^{4}$ (1) Salman Khan ${ }^{2}(\mathbb{0}$
}

Received: 15 April 2021 / Accepted: 10 September 2021

(c) The Author(s), under exclusive licence to Springer Nature B.V. 2021

\begin{abstract}
Background Neuropathic pain is a chronic pain state that negatively impacts the quality of life. Currently, available therapies for the treatment of neuropathic pain often lack efficacy and tolerability. Therefore, the search for novel drugs is crucial to obtain treatments that effectively suppress neuropathic pain.

Objectives The present study was undertaken to investigate the antinociceptive properties of (1,4-bis-(diphenylphosphino) butane) palladium (II) chloride monohydrate (Compound 1) in a paclitaxel (PTX)-induced neuropathic pain model.

Methods Initially, behavioral tests such as mechanical and cold allodynia as well as thermal and tail immersion hyperalgesia were performed to investigate the antinociceptive potential of Compound $1(5$ and $10 \mathrm{mg} / \mathrm{kg}, \mathrm{b} . \mathrm{w})$. RT-PCR was performed to determine the effect of Compound 1 on the mRNA expression level of inducible nitric oxide synthase (iNOS), cyclooxygenase-2 (COX-2), and proinflammatory cytokines such as tumor necrosis factor-alpha (TNF)- $\alpha$, interleukin (IL)-1 $\beta$, and IL-6. In addition, antioxidant protein, nitric oxide (NO), and malondialdehyde (MDA) levels were also determined.

Results The results demonstrated that once-daily dosing of Compound 1 significantly suppressed the PTX-induced behavioral pain responses dose-dependently. The mRNA gene expressions of iNOS, COX-2, and inflammatory cytokines were markedly reduced by Compound 1. Furthermore, it enhanced the level of antioxidant enzymes and lowered the level of MDA and NO production.

Conclusion These findings suggest that the antinociceptive potential of Compound 1 in the PTX-induced neuropathic pain model is via suppression of oxidative stress and inflammation. Thus, Compound 1 might be a potential candidate for the therapeutic management of PTX induced neuropathic pain.
\end{abstract}

Keywords Allodynia $\cdot$ Hyperalgesia $\cdot$ iNOS $\cdot \mathrm{COX}-2 \cdot$ Inflammatory mediators $\cdot$ Oxidative stress

Zia ur Rehman

zrehman@qau.edu.pk

Salman Khan

skhan@qau.edu.pk

1 Department of Pharmacology and Pharmacotherapy, Faculty of Medicine, University of Szeged, Szeged, Hungary

2 Pharmacological Sciences Research Lab, Department of Pharmacy, Faculty of Biological Sciences, Quaid-I-Azam University, Islamabad, Pakistan

3 Department of Pharmacy, University of Peshawar, Peshawar, Pakistan

4 Department of Chemistry, Quaid-I-Azam University, Islamabad 45320, Pakistan

5 Department of Chemistry, University of Science and Technology, KPK, Bannu 28100, Pakistan

\section{Introduction}

Neuropathic pain occurs due to the damage of peripheral or central nerves [1,2]. It is generally characterized by extreme sensitivity to non-noxious stimuli (allodynia) and noxious stimuli (hyperalgesia). Neuropathic pain arises due to trauma, disease, and chemotherapeutic drugs [2,3]. Macrophages are a vital component of the immune system and play pivotal roles in the regulation of inflammatory responses [4]. Macrophages are implicated in the pathogenesis of neuropathic pain [5]. Following nerve injury, resident macrophages initiate inflammatory responses and elicit long-lasting neuroinflammation through the recruitment of circulating leukocytes to the site of injury [4]. At the periphery, activated macrophages secrete cytokines/ chemokines such as TNF- $\alpha$ (tumor necrosis factor- $\alpha$ ) and 
interleukins (IL-1 $\beta$, IL-6). Cytokines and chemokines secreted by macrophages are potential mediators of pain hypersensitivity in neuropathic pain [5]. Paclitaxel (PTX) is a well-known chemotherapeutic agent that is widely used for the treatment of breast, ovarian, lung, and bladder cancers [6]. However, treatment with PTX may result in peripheral neuropathy that exists for several months even after discontinuation of therapy [7]. Peripheral neuropathy is a major reason for reduction of dose or even cessation of chemotherapy and therefore, results in a great impact on the survival of cancer patients [8].

Numerous studies have revealed that inflammatory responses in the spinal cord result in the development of chemotherapy-induced peripheral neuropathy $[9,10]$. It is well recognized that pro-inflammatory mediators are released by activated glial cells in the spinal cord which intensifies the sensitivity and excitability of the neuron [11]. Several lines of evidence have indicated that COX-2 (an inducible enzyme) and iNOS are associated with the induction and progression of neuropathic pain [12]. Peripheral nerve injury elevates the expression of $\mathrm{COX}-2$ in the spinal cord which facilitates inflammation and pain hypersensitivity [13]. In addition, oxidative stress plays a crucial role in the pathogenesis of neuropathic pain [14].

Currently, treatment for neuropathic pain includes NSAIDs, morphine, anti-convulsant, and anti-depressants drug. However, these treatments have been associated with a wide spectrum of adverse effects [2]. The compounds containing structural palladium thiocarbamate have been reported for various anti-inflammatory and analgesic properties [15]. Pronounced pharmacological effects with a better safety profile have been considered by designing and synthesizing palladium-containing complexes [16]. The dithiocarbamates have also been reported for promising activities against oxidative stress and inflammatory cytokine [17]. It has been noted that dithiocarbamates structural analogs exhibit antiarthritic activities via inhibition of NF- $\mathrm{KB}$ and COX-2 signaling [18]. The characterization of Compound 1 has recognized it to be an efficient drug moiety. Earlier results have indicated that Compound 1 has suppressed the complete Freund's adjuvant (CFA)-, carrageenan-, histamine-, and serotonin-induced nociception [19]. Thus, bearing in mind the above-mentioned results, we decided to further explore the neuroprotective property of Compound 1 against PTX-induced neuropathic pain model.

\section{Materials and methods}

\section{Chemicals and reagents}

The DNA Extraction Kit (Novel Genomic DNA Mini Kit), TRI-Reagent (Bioshop, Canada), cDNA Synthesis Kit (ABM, Canada), PCR Primers (Macrogen Korea), PCR
Master Mix (Thermo Fisher Scientific US). PTX, Compound 1, gabapentin (GBP), Griess Reagent, 1-chloro-2,4-dinitrobenzene (CDNB), dithio-nitrobenzoic acid (DTNB), hydrogen peroxide ( $\mathrm{H} 2 \mathrm{O} 2)$, and all the other chemicals used were obtained from Sigma (USA). Drugs were dissolved in dimethyl sulfoxide (DMSO 2\%) and diluted with normal saline up to the final volume.

\section{Synthesis of compound 1}

Compound 1 (1,4-bis-(diphenylphosphino) butane) palladium (II)chloride monohydrate) was synthesized according to the literature procedure [19]. Briefly, it was prepared by simultaneous and dropwise addition of 1,4-bis(diphenylphosphino) butane $(0.426 \mathrm{~g}, 1 \mathrm{mmol})$ in acetone $(40 \mathrm{~mL})$ and sodium 4-benzylpiperidine-1-carbodithioate $(0.273 \mathrm{~g}, 1 \mathrm{mmol})$ in methanol $(25 \mathrm{~mL})$ to a methanolic suspension of palladium (II) chloride $(0.177 \mathrm{~g}, 1 \mathrm{mmol})$. The reaction mixture was refluxed for $6 \mathrm{~h}$ with constant stirring. It was then filtered, and the filtrate was rotary evaporated. The product was soluble in acetone, chloroform, methanol, and dimethylsulfoxide.

\section{Animals and experimental design}

Sprague-Dawley female rats (200-250 g) were used in the present study. The animals were obtained from the NIH Islamabad, Pakistan. All the rats were accommodated in standard environmental conditions $\left(22 \pm 1{ }^{\circ} \mathrm{C}, 52 \pm 3 \%\right.$ humidity) and a light/dark period of 12-h with unrestricted access to food and water.

\section{Induction of neuropathic pain and dosing}

PTX (4 mg/kg) was injected intraperitoneally (i.p.) in volumes of $1 \mathrm{ml}$ per kg, 4 times a week (on days 1, 3, 5, and 7) [20]. PTX dose selection was selected based on a previous report [20]. Compound 1 (5 and $10 \mathrm{mg}$ ) was administered once daily for 8 days. The doses of Compound 1 were selected based on its previously reported therapeutic activities in animal models of arthritis [19]. Previously, in the first set of experiments, different doses of Compound 1 were screened to select an optimum and effective dose. Compound 1 was tested at a dose level of $0.1,1$, and $10 \mathrm{mg} /$ $\mathrm{kg}$. It exhibited optimum anti-inflammatory and analgesic effects at the doses of 5 and $10 \mathrm{mg} / \mathrm{kg}$ of body weight in mice [19]. The schedule of behavioral experimentations was on day 0 i.e., before first drug administration and then on every other day of PTX administration i.e., on days 2, 4, 6, and 8 after $1 \mathrm{~h}$ of treatment. Samples were collected after all the rats were sacrificed from each group on the final day of the experiment (Fig. 1). 


\section{Animal's models}

Compound 1 was tested in a PTX-induced neuropathic pain model. All the animals were allotted in normal, negative (PTX-induced), positive (GBP) and treatment groups ( 5 and $10 \mathrm{mg} / \mathrm{kg}$ of Compound 1) with 6 animals in each group. Behavioral testing was carried out by an experimenter blinded to the treatment groups.

\section{PTX-induced model}

Rats were distributed into five groups with six rats per group:

Group 1: Normal control.

Group 2: PTX-induced (4 mg/kg, i.p).

Group 3: GBP $75 \mathrm{mg} / \mathrm{kg}$ (once daily for 8 days, i.p).

Group 4: Compound 1 (5 mg/kg, once daily for 8 days, i.p).

Group 5: Compound 1 (10 mg/kg, once daily for 8 days, i.p).

\section{Behavioral experiments}

\section{Evaluation of static mechanical allodynia in PTX-induced rats}

The anti-allodynic effect of Compound 1 was measured at various intervals (i.e. on days $0,2,4,6$, and 8) using Von Frey (VF) filaments as described previously [21-23].

\section{Evaluation of cold allodynia (acetone test)}

Compound 1 ( 5 and $10 \mathrm{mg} / \mathrm{kg}$ ) was investigated against PTX-induced cold pain (allodynia) by performing an acetone test as described previously [24, 25]. In short, rats were habituated for $15 \mathrm{~min}$ in a transparent plastic box with a wire mesh floor. After that, a drop $(0.05 \mathrm{~mL})$ of acetone was put on the rat's plantar skin surface using a syringe. The behaviors of rats (i.e. time taken by a rat while flinching, licking, withdrawing, and biting of hind paw) after the acetone spray was examined within $15 \mathrm{~s}$ and measured as paw withdrawal duration (PWD). A comparative increase in PWD (s) is considered to be a sign of neuropathic pain. Acetone was ejected three times on each hind paw and their average PWD was calculated for each rat.

\section{Evaluation of thermal hyperalgesia}

To evaluate further the analgesic effect of Compound 1 in PTX-induced rats, a hot plate test was performed according to the previously reported protocols [26-28]. The once-daily dosing of Compound 1 was examined for antihyperalgesic effect on days $0,2,4,6$, and 8 .

\section{Evaluation of tail-flick latency (hot)}

A hot tail immersion assay was performed to evaluate the central anti-nociceptive activity of Compound 1 as described previously $[29,30]$. Briefly, the rat's tail was immersed into $52{ }^{\circ} \mathrm{C}$ water for $15 \mathrm{~s}$ (maximum), and the latency when the rat withdrew the tail was noted. The time taken by a rat to flick its tail from hot water was recorded as tail withdrawal latency (TWL). Each rat was exposed to three trials with a break of $5 \mathrm{~min}$ in between. The average of 3 trials was calculated as latency for each rat.

\section{Evaluation of tail-flick latency (cold)}

The cold-water tail-flick assay was performed to evaluate the cold hyperalgesia and antihyperalgesic effect of Compound 1 by dipping the lower half of the rat's tail into a beaker filled with cold water $\left(0-4{ }^{\circ} \mathrm{C}\right)[29,31]$. After dipping the tail, the TWL was measured with a $15 \mathrm{~s}$ cut-off time. The test was studied 3 times at 5 min intervals to avoid tissue damage and the average was calculated. The antihyperalgesic effect was represented by the average of each latency (i.e. the comparatively longer latency was considered the antihyperalgesic effect) [32].

Experimental design

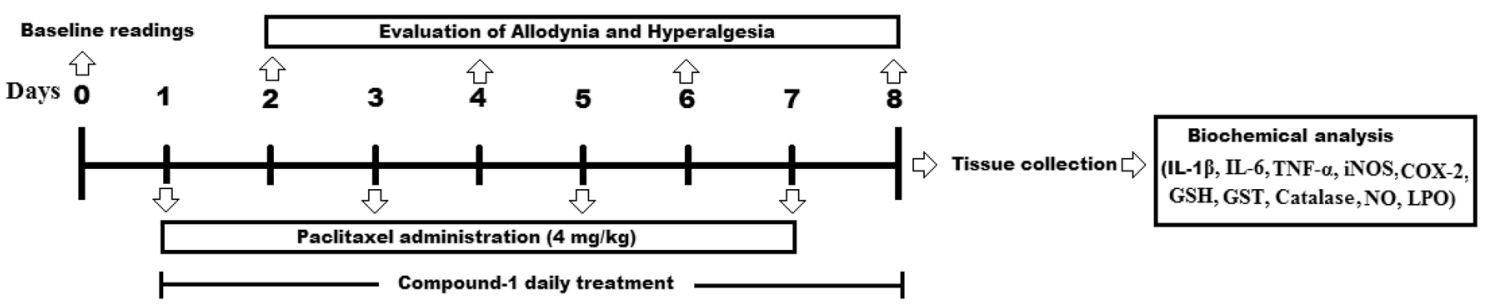

Fig. 1 A schematic representation of overall study plan 


\section{Evaluation of motor performances}

Assessment of Compound 1 treatment on motor performances of rats was evaluated using a rotarod apparatus, which was rotating at a speed up to $40 \mathrm{rpm}$ for $5 \mathrm{~min}$. Before starting the experiment, all the rats were trained for 4 days by placing them on a rotating drum with a maximum speed of $40 \mathrm{rpm}$ as described previously [33, 34]. The rats which did not endure walking on the rotarod for more than 2 min were excluded. After that baseline trials were performed before any drug administration to start the experiment. Each rat underwent 3 trials. The latency of time to fall and the falling frequency over a 5-min period were measured. The same trials were repeated on days 2 , 4, 6, and $8,1 \mathrm{~h}$ after treatment with Compound 1 or GBP.

\section{Biochemical assays}

\section{RNA extraction and RT-PCR}

Total RNA was extracted from the L4-6 spinal cord segment of rats according to the manufacturer's protocol using the TRI-reagent and the purity of the total RNA was determined using a UV spectrophotometer. Total RNA was transcribed to cDNA using a cDNA synthesis kit. The expressions of targeted genes like TNF- $\alpha$, IL-1 $\beta$, IL-6, iNOS, and COX- 2 were determined (Table. 1 ). $\beta$-actin was incorporated as the housekeeping gene. Amplified products were isolated by the use of $1.5 \%$ agarose gel electrophoresis and visualized through a UV trans-illuminator. The expression level (A.U) was calculated [11,35-38].

\section{Nitric oxide (NO) determination}

The production of NO in the spinal tissue was quantified using the Griess reagent assay according to the previously reported protocols [39-41].

\section{Determination of antioxidant enzymes}

The levels of antioxidants such as GSH, GST, and catalase were measured according to the previously reported method [42, 43].

\section{Lipid peroxidation assay (LPO)}

LPO was estimated by measuring the MDA concentration in spinal tissue according to the previously reported
Table 1 The sequences of PCR primers

\begin{tabular}{ll}
\hline Primer & Sequence \\
\hline IL-1 $\beta$ & F- 5'-TGATGACGACCTGCTAGTGTG-3 \\
& R- 5'- TCCATTGAGGTGGAGAGCTT-3 \\
IL-6 & F- 5'-GGAGTTTGTGAAGAACAACT 3 \\
& R- 5'- CTAGGGTTTCAGTATTGCTC-3 \\
TNF- $\alpha$ & F- 5'- ATGAGCACAGAAAGCATGATC-3' \\
& R- 5'-TACAGGCTTGTCACTCGAATT-3' \\
iNOS & F- 5'-CACCACCCTCCTTGTTCAAC-3' \\
& R; 5'-CAATCCACAACTCGCTCCAA-3' \\
COX-2 & F- 5'-TGTATGCTACCATCTGGCTTCGG-3' \\
& R- 5'-GTTTGGAACAGTCGCTCGTCATC-3' \\
$\beta$-actin & F- 5'- CGTTGACATCCGTAAAGACCTC-3'; \\
& R- 5'-TAGGAGCCA GGGCAGTAATCT-3' \\
\hline
\end{tabular}

method [44-46]. A microplate reader was used to measure the absorbance at $535 \mathrm{~nm}$.

\section{Pharmacokinetics and toxicokinetic analysis}

An in-silico analysis was performed to determine the pharmacokinetic behavior of Compound 1 . The various pharmacokinetics factors that were assessed include absorption, distribution, metabolism, excretion, the volume of distribution, and plasma protein binding. The pharmacokinetic parameters were analyzed using Swiss target prediction and pK-CSM online server as reported previously [47]. Similarly, the toxicokinetic analysis was performed to assess the harmful effect of Compound 1 against animals, tissue, and micro-organisms, and the maximum tolerated dose was established using online computational tools [47].

\section{Statistical analysis}

Data expressed as the mean \pm SD. Two-way ANOVA was applied followed by Bonferroni's post hoc test for the assessment of statistical significance amongst various treated groups. Statistical analysis of the data was performed using Sigma-plot version 12.5. $p$ " value $<0.05$ was considered to be statistically significant.

\section{Results}

\section{Effect of Compound 1 on static mechanical allodynia}

Allodynia produced by administration of PTX was considerably $(p<0.001)$ inhibited by treatment with Compound 1 and GBP in a dose-dependent manner. The antiallodynic effect was indicated by increased paw withdrawal threshold (PWT) by treatment with Compound 1 when evaluated on 
the 2nd, 4th, 6th, and $8^{\text {th }}$ day. The PWT (g) was remarkably high in the treatment groups as compared to the PTX group (Fig. 2a).

\section{Effect of Compound 1 on cold allodynia}

Cold allodynia in PTX-treated rats was significantly $(p<0.001)$ high as compared to the normal control groups as evident from the comparative rise in PWD (s). However, this increase in PWD was reduced by treatment with Compound 1 and GBP (Fig. 2b).

\section{Effect of Compound 1 on thermal hyperalgesia}

Treatment with Compound 1 markedly $(p<0.001)$ lowered the hyperalgesic responses (paw licking and jumping) dosedependently in PTX-treated rats as denoted by increased paw withdrawal latency (PWL) in treatment groups (Fig. 2c).

\section{Effect of Compound 1 on tail hot hyperalgesia}

The hot tail immersion nociception is indicative of central nociception. Rats treated with PTX exhibited shortened TWL (s) as compared to the normal control. However, Compound 1 treatment markedly $(p<0.001)$ increased TWL (Fig. 2d).

\section{Effect of Compound 1 on tail cold hyperalgesia}

In PTX-treated rats, TWL was significantly reduced than normal animals indicating cold hyperalgesia. Compound 1 daily treatment remarkably $(p<0.001)$ elevated this reduction in TWL showing its anti-neuropathic pain potential (Fig. 2e).

\section{Effect of Compound 1 on motor activity}

Motor coordination was assessed through a rotarod test. No abnormal effect was observed on motor activity after the treatment with Compound 1. However, GBP exhibited a significant motor deficit indicated from low rotarod latency time (sec) (Fig. 2f).

\section{Effect of Compound 1 on inflammatory cytokines}

The mRNA level of inflammatory cytokines in spinal cord tissues was analyzed using RT-PCR. A significant $(p<0.001)$ induction in the expression of these cytokines was observed in the PTX-treated group. However, treatment with Compound 1 significantly $(p<0.001)$ lowered the expression of these cytokines as compared to the negative control group (Fig. 3b-d).
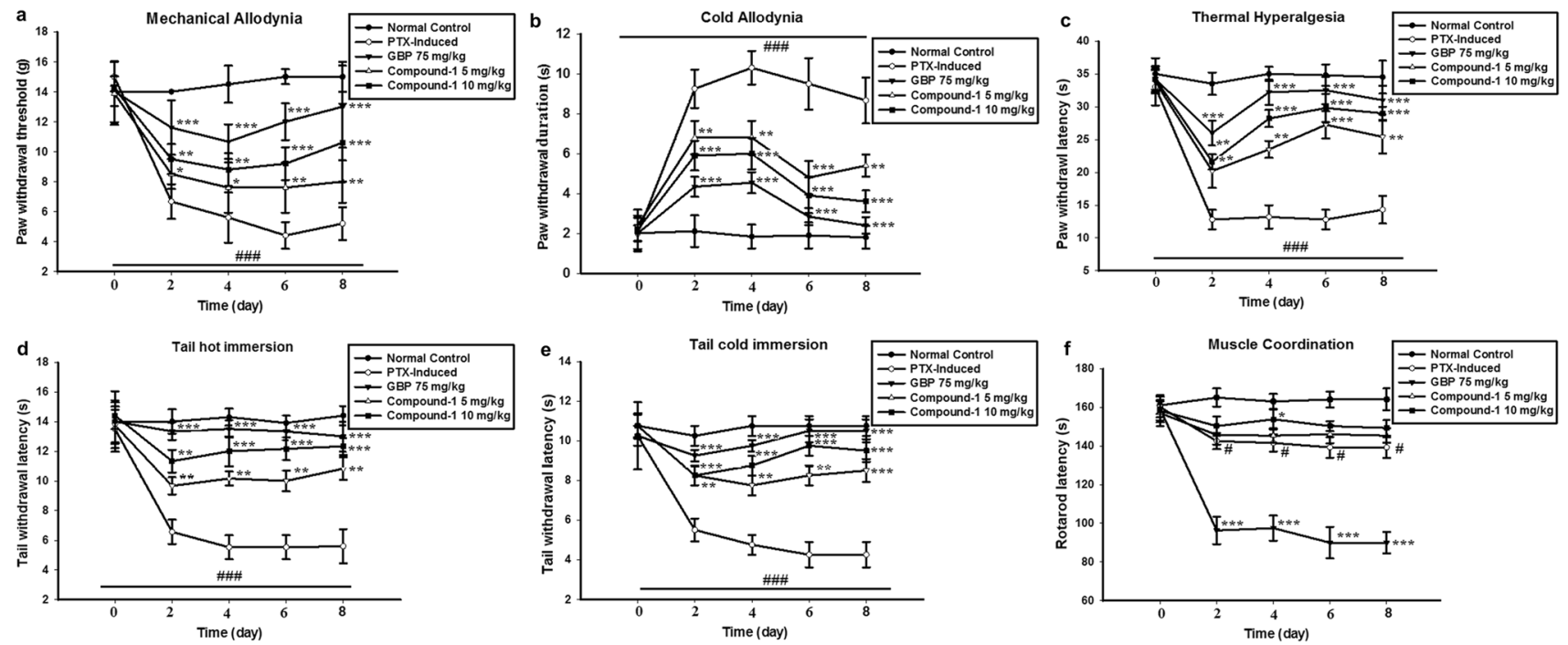

Fig. 2 Dose-dependent effect of Compound 1 in doses of 5 and $10 \mathrm{mg} / \mathrm{kg}$ in PTX-induced rats. Compound 1 pretreatment inhibited PTX-induced (a) mechanical allodynia (b) cold allodynia (c) thermal hyperalgesia (d) tail thermal hyperalgesia (e) tail cold hyperalgesia and (f) motor activity. The data is displayed as the mean $(n=6) \pm$ SD.
ANOVA followed by a post hoc Bonferroni test was applied for comparing statistical differences between groups. ${ }^{*} p \leq 0.05,{ }^{*} p \leq 0.01$, and $* * * p \leq 0.001$ represent a statistically significant difference from the PTX-induced group. (\#\#\#) indicates comparison to the normal control group 


\section{Effect of Compound 1 on iNOS and COX-2 mRNA expression levels}

To further determine the inhibitory effect of Compound 1 on inflammatory mediators, we evaluated the mRNA expression level of iNOS and COX-2 in the spinal cord. There was an up-regulation of iNOS and COX-2 mRNA expression after neuropathic pain induction which was significantly $(p<0.001)$ inhibited by treatment with Compound 1 (Fig. 3e, f).

\section{Effect of Compound 1 on antioxidants}

The levels of antioxidants were significantly $(p<0.001)$ reduced in the PTX-treated group. However, Compound 1 increased the level of these antioxidant proteins as shown in (Table. 2).

\section{Effect of Compound 1 on nitric oxide (NO) production}

The NO production was significantly $(p<0.001)$ increased in the PTX-treated group. Treatment with Compound 1 remarkably $(p<0.001)$ inhibited the PTX-induced NO production (Table. 2).

\section{Effect of Compound 1 on LPO}

PTX administration significantly $(p<0.001)$ elevated the MDA level. Compound 1 treatment showed a marked $(p<0.001)$ decrease in MDA level as compared PTX-treated group (Table. 2).

\section{Pharmacokinetics and toxicokinetic analysis}

The pharmacokinetic analysis of the compounds showed variable pharmacokinetic properties using online pKCSM software (http://biosig.unimelb.edu.au/pkcsm/prediction).
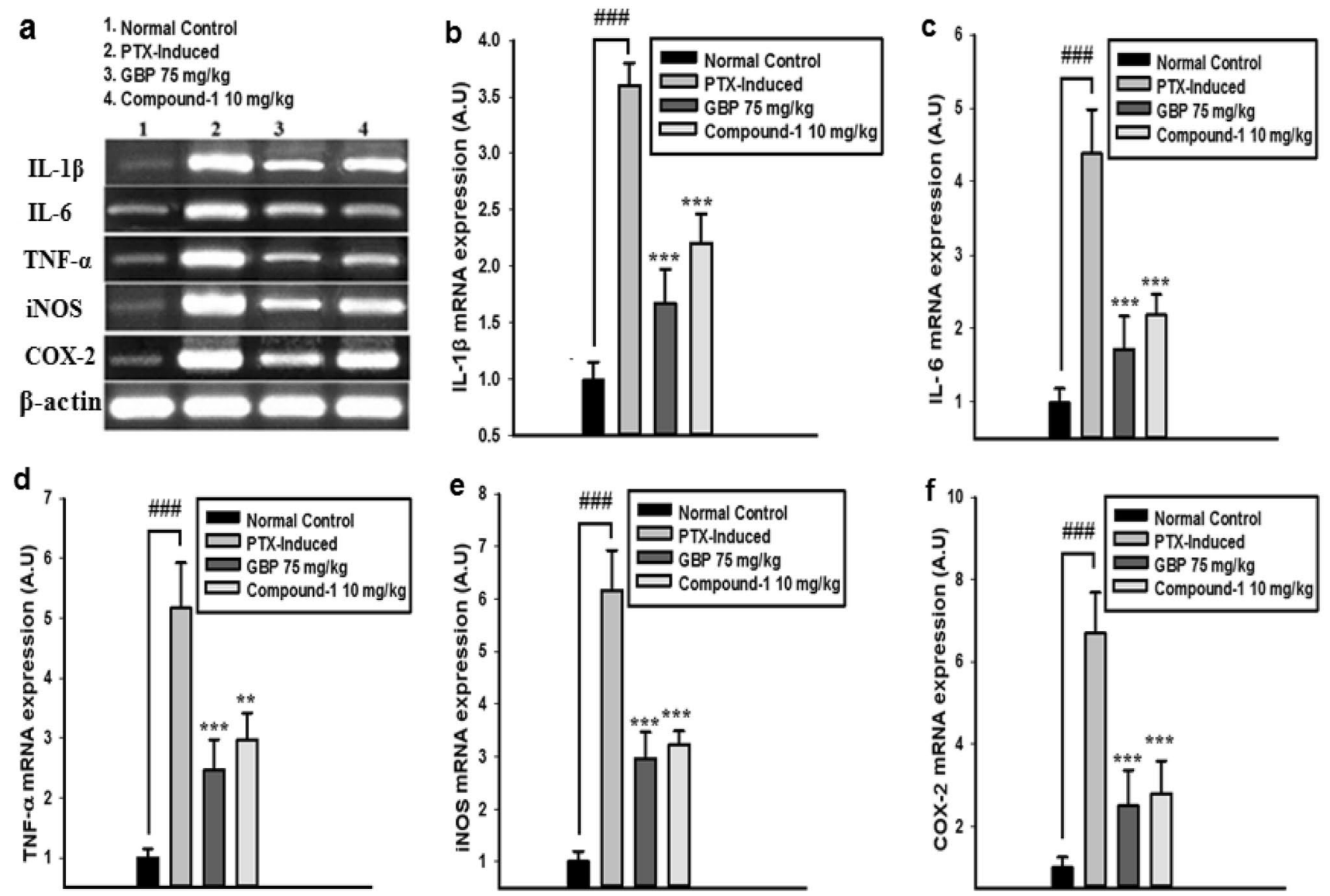

Fig. 3 a Effect of Compound 1 treatment on mRNA expression level of (b) IL-1 $\beta$ (c) IL-6 (d) TNF- $\alpha$ (e) iNOS and (f) COX-2. The results are shown in a relatively arbitrary unit (A.U). The data is displayed as the mean $(n=6) \pm S D$. ANOVA followed by a post hoc Bonferroni test was applied for comparing statistical differences between groups. ${ }^{*} p \leq 0.05,{ }^{*} p \leq 0.01$, and $* * * p \leq 0.001$ represent a statistically significant difference from the PTX-induced group. (\#\#\#) indicates comparison to the normal control group 
Table 2 Effect of Compound 1 on antioxidants, $\mathrm{LPO}$ and $\mathrm{NO}$ production in PTX-induced rat's spinal tissue

\begin{tabular}{lrrll}
\hline Parameters & Normal control & \multicolumn{1}{l}{ PTX } & GBP $(75 \mathrm{mg} / \mathrm{kg})$ & Compound 1 $(10 \mathrm{mg} / \mathrm{kg})$ \\
\hline GSH concentration $(\mu \mathrm{M})$ & $14.47 \pm 0.39$ & $3.45 \pm 0.28^{\# \# \#}$ & $11.42 \pm 0.39^{* * *}$ & $10.90 \pm 0.36^{* * *}$ \\
GST concentration $(\mu \mathrm{M})$ & $38.92 \pm 1.27$ & $12.43 \pm 1.38^{\# \# \#}$ & $36.70 \pm 1.14^{* * *}$ & $36.25 \pm 1.66^{* * *}$ \\
Catalase activity (unit/ $\mu \mathrm{L})$ & $11.6 \pm 0.17$ & $3.88 \pm 0.09^{\# \# \#}$ & $11.16 \pm 0.09^{* * *}$ & $11.03 \pm 0.14^{* * *}$ \\
MDA level $(\%)$ & $10.68 \pm 0.30$ & $100 \pm 2.86^{\# \# \#}$ & $16.89 \pm 1.68^{* * *}$ & $18.01 \pm 0.68^{* * *}$ \\
NO production $(\mu \mathrm{M})$ & $7.61 \pm 0.51$ & $56.06 \pm 6.56^{\# \# \#}$ & $11.29 \pm 1.90^{* * *}$ & $11.64 \pm 1.34^{* * *}$ \\
\hline
\end{tabular}

The data is presented as the mean $(n=6) \pm S D$

(\#\#\#) denotes comparison to normal control group

*** $p$ denotes comparison to PTX-induced group

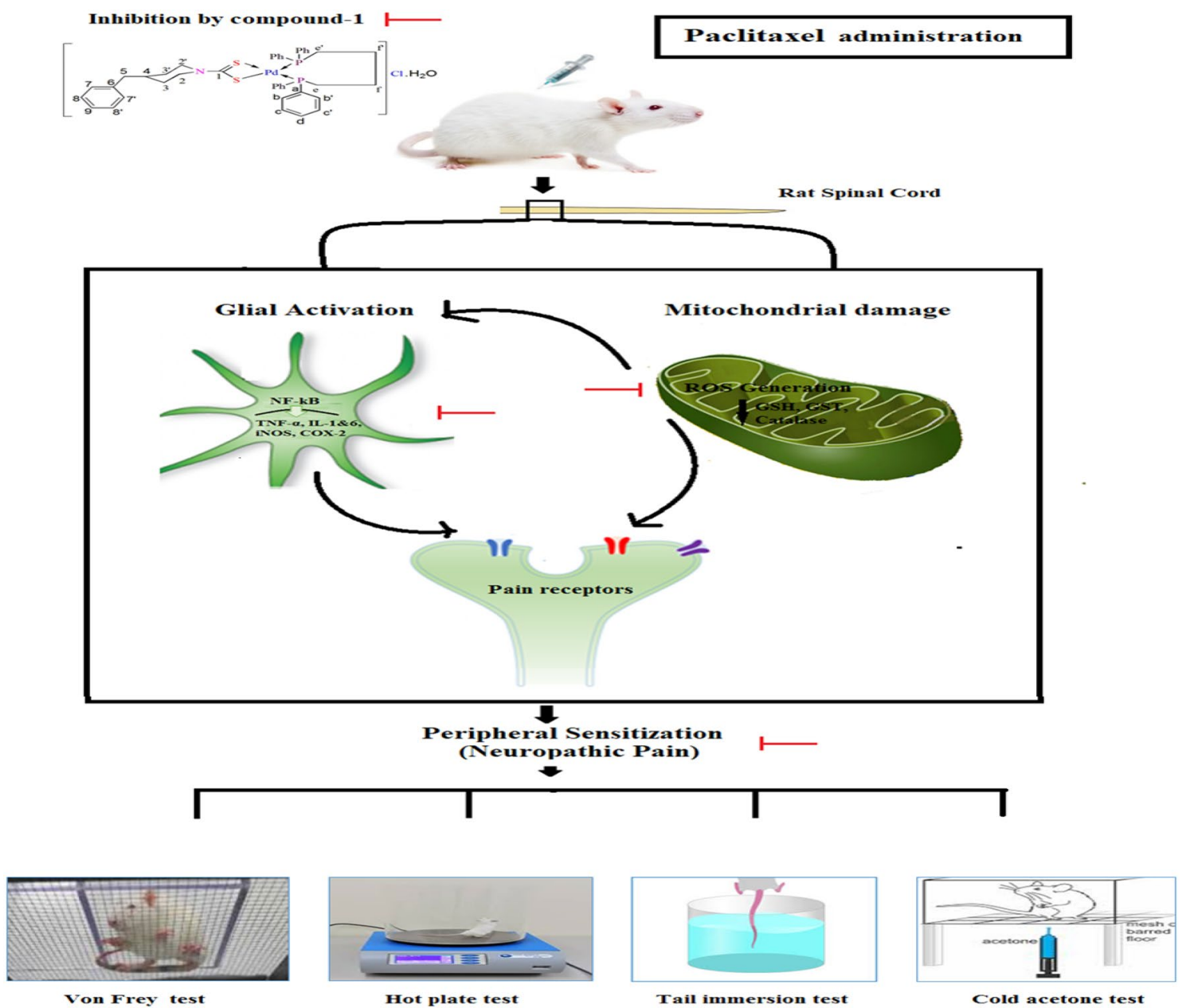

Fig. 4 Graphical abstract representing anti-neuropathic pain activity of Compound 1 in PTX-induced neuropathic pain model

The various properties that were predicted include druglike properties, physicochemical properties, lipophilicity, water solubility, and pharmacokinetic properties. Similarly, the toxicokinetic analysis of the Compound 1 showed a good safety profile as shown in Table 3 .

\section{Discussion}

PTX is an effective chemotherapeutic drug indicated in the treatment of breast, ovarian, lung, and bladder cancers. However, PTX-induced peripheral neuropathy is a 
Table 3 Pharmacokinetic and toxicokinetic analysis of the Compound 1

\begin{tabular}{|c|c|c|c|c|c|c|c|c|c|}
\hline \multirow[t]{2}{*}{ Name } & \multicolumn{4}{|l|}{ Absorption } & \multicolumn{3}{|l|}{ Distribution } & \multirow[b]{2}{*}{$\begin{array}{l}\text { Metabolism } \\
\text { CYP } \\
\text { 2D6 3A4 } \\
\text { 1A2 2C19 } \\
\text { 2C9 2D6 } \\
\text { 3A4 } \\
\text { Substrate } \\
\text { Inhibitor } \\
\text { Categorical } \\
\text { (Yes/No) }\end{array}$} & \multirow{2}{*}{$\begin{array}{l}\text { Excretion } \\
\begin{array}{l}\text { Numeric } \\
(\operatorname{log~ml} / \mathrm{min} \\
\mathrm{kg})\end{array}\end{array}$} \\
\hline & $\begin{array}{l}\text { Water solu- } \\
\text { bility (log } \\
\mathrm{mol} / \mathrm{l})\end{array}$ & Caco2 Cell & $\begin{array}{l}\text { Intestinal } \\
\text { absorption }\end{array}$ & $\begin{array}{l}\text { P-gp } \\
\text { Substrate } \\
\text { Cat- } \\
\text { egorical } \\
\text { (Yes/ } \\
\text { No) }\end{array}$ & $\begin{array}{l}\text { BBB } \\
\text { permeability } \\
(\log B B)\end{array}$ & $\begin{array}{l}\text { Fraction } \\
\text { Unbound } \\
\text { Numeric } \\
(\mathrm{Fu})\end{array}$ & $\begin{array}{l}\text { CNS VDss } \\
\text { Numeric } \\
\text { (log PS) } \\
\log \mathrm{L} / \mathrm{kg}\end{array}$ & & \\
\hline Compound 1 & -5.34 & 1.009 & 94.37 & Yes & 1.445 & 0.300 & $\begin{array}{r}-0.056 \\
-0.753\end{array}$ & $\begin{array}{l}\text { No Yes No } \\
\text { No No No } \\
\text { No }\end{array}$ & -1.109 \\
\hline $\begin{array}{l}\text { Molecular } \\
\text { properties }\end{array}$ & $\begin{array}{l}\text { Molecular } \\
\text { weight }\end{array}$ & & Logp & & $\begin{array}{l}\text { Rotatable } \\
\text { bonds }\end{array}$ & & Acceptors & Donors & $\begin{array}{l}\text { Surface } \\
\text { area }\end{array}$ \\
\hline Compound 1 & 785.328 & & 8.918 & & 6 & & 2 & 0 & 290.311 \\
\hline Toxicity & $\begin{array}{l}\text { Ames toxic- } \\
\text { ity }\end{array}$ & Max dose & $\begin{array}{l}\text { hERG I } \\
\text { inhibitor }\end{array}$ & $\begin{array}{l}\text { hERG II } \\
\text { inhibitor }\end{array}$ & $\begin{array}{l}\text { Oral toxic- } \\
\text { ity acute } \\
\text { (LD50) }\end{array}$ & $\begin{array}{l}\text { Oral chronic } \\
\text { toxicity }\end{array}$ & $\begin{array}{l}\text { Hepatotoxic- } \\
\text { ity }\end{array}$ & $\begin{array}{l}\text { Skin sensi- } \\
\text { tivity T. } \\
\text { Pyriformis } \\
\text { toxicity }\end{array}$ & $\begin{array}{l}\text { Minnow } \\
\text { toxicity }\end{array}$ \\
\hline Compound 1 & No & 0.45 & No & Yes & 3.263 & -0.367 & No & No 0.285 & 3.039 \\
\hline
\end{tabular}

major adverse effect and causes premature termination of cancer therapy. This adverse effect may persist up to several months even after discontinuation of therapy [7]. Current therapies have been associated with a wide spectrum of adverse effects that limit their satisfactory clinical use. It is important to identify novel drugs that effectively suppress neuropathic pain without attenuating their anticancer effects [2]. Numerous studies have shown the role of inflammatory cytokines, NO, COX-2, and oxidative stress in the initiation and maintenance of neuropathic pain [48-50]. Therefore, the drug that inhibits the release of inflammatory mediators might be the potential candidate to treat neuropathic pain. Previous findings have demonstrated that Compound 1 exhibited remarkable antiinflammatory and analgesic activities by suppressing the inflammatory mediator's production. Therefore, keeping in view the previous promising therapeutic activities, Compound 1 was further evaluated as an anti-neuropathic pain agent in the present study.

PTX significantly induced allodynia and hyperalgesia by increasing the rat's plantar sensitivity to VF filaments, hot, and cold stimuli. The neuropathic pain produced by PTX administration was measured in the form of various pain parameters such as decreasing PWT, PWL, TWL, and increase in PWD [29]. However, these parameters of nociception were significantly improved by daily treatment with Compound 1 and GBP. Results from all the behavioral responses demonstrated the significant antiallodynic and antihyperalgesic activities of Compound 1. Furthermore, Compound 1 did not show any deteriorating effect on motor activity.
Inflammatory cytokines play a crucial role in neuropathic pain by increased hypersensitivity [1] and lead to excessive nociceptive transmission in the spinal cord [51]. The chemical mediators secreted all over the inflammatory process sensitizes the nociceptors that produce pain hypersensitivity resulting in hyperalgesia and allodynia [19]. PTX has been revealed to induce the expression of proinflammatory cytokines, such as IL-1 and TNF- $\alpha$, in the rat spinal cord [52]. In the present study, PTX treatment considerably increased the expression of inflammatory cytokines in spinal tissues. These inflammatory cytokines were remarkably reduced by Compound 1 . Similarly, the iNOS and COX-2 expression levels were also measured. The present study showed that Compound 1significantly suppressed iNOS and COX-2 expressions as well as NO production.

It is well known that oxidative stress plays a vital role in neuropathic pain [2]. In the present study, PTX administration significantly lowered the level of endogenous antioxidants i.e., GSH, GST, and catalase, respectively. The results demonstrated that Compound 1 exhibited a protective role against the PTX-induced oxidative stress by enhancing the level of antioxidants in spinal tissues while decreasing the MDA level. Additionally, the pharmacokinetic analysis using pK-CSM software showed that Compound 1 exhibits high GIT absorption, BBB permeability, and interaction with the cytochrome p450 system, and partial risk of toxicity. 


\section{Conclusion}

In summary, the present study revealed that the compound possesses significant antiallodynic and antihyperalgesic activities in the well-known PTX-induced neuropathic pain model. Compound 1 markedly suppressed the levels of proinflammatory cytokines, iNOS, COX-2, and oxidative stress (Fig. 4). The pharmacokinetic analysis showed that Compound 1 exhibits good ADME properties. We suggest from the present study that Compound 1 might be a useful candidate for the treatment of neuropathic pain. Additional research is needed to confirm these results.

Authors' contributions $\mathrm{MN}$, RU designed and performed research including behavioral and biochemical assays. SZK and ZR synthesized the compound. AK helped in behavioral and biochemical assays. $\mathrm{AK}$ and $\mathrm{MN}$ write the original manuscript. MN, AK, BS, AUK, and SK analyzed the data. SK supervised the project. All authors read and approved the final manuscript.

Data Availability The datasets generated during and/or analyzed during the current study are available from the corresponding author on reasonable request.

\section{Declarations}

Conflict of interest The authors declare they have no conflict of interest.

Ethical approval "QAU guidelines for animal's care" Islamabad were followed for the overall experiments involving animals. QAU, Islamabad Bioethical Committee (Approval No: BEC-FBS-QAU 2017-59) approved the study. Maximum care was assured to minimize harm to animals.

\section{Consent for publication N/A}

\section{References}

1. Khan S et al (2016) Attenuation of neuropathic pain and neuroinflammatory responses by a pyranocoumarin derivative, anomalin in animal and cellular models. Eur J Pharmacol 774:95-104

2. Khan A et al (2021) Suppression of TRPV1/TRPM8/P2Y nociceptors by withametelin via downregulating MAPK signaling in mouse model of vincristine-induced neuropathic pain. IJMS 22(11):6084

3. Khan A et al (2021) 7 $\beta$-(3-Ethyl-cis-crotonoyloxy)-1 $\alpha-(2-$ methylbutyryloxy)-3, 14-dehydro-Z notonipetranone attenuates neuropathic pain by suppressing oxidative stress. Inflam ProApoptotic Protein Expressions 26(1):181

4. Kiguchi $\mathrm{N}$ et al (2017) Pharmacological regulation of neuropathic pain driven by inflammatory macrophages 18(11):2296

5. Ristoiu VJL (2013) Contribution of macrophages to peripheral neuropathic pain pathogenesis. Life Sci 93(23):870-881

6. Manjavachi MN et al (2019) Spinal blockage of CXCL1 and its receptor CXCR2 inhibits paclitaxel-induced peripheral neuropathy in mice. Neuropharmacology 151:136-143
7. Seretny M et al (2014) Incidence, prevalence, and predictors of chemotherapy-induced peripheral neuropathy: a systematic review and meta-analysis. Pain 155(12):2461-2470

8. Starobova H, Vetter I (2017) Pathophysiology of chemotherapyinduced peripheral neuropathy. Front Mol Neurosci 10:174

9. Janes $\mathrm{K}$ et al (2015) Spinal neuroimmune activation is independent of T-cell infiltration and attenuated by A3 adenosine receptor agonists in a model of oxaliplatin-induced peripheral neuropathy. Brain Behav Immun 44:91-99

10. Makker PG et al (2017) Characterisation of immune and neuroinflammatory changes associated with chemotherapy-induced peripheral neuropathy. PLoS ONE 12(1):e170814

11. Kanda $\mathrm{H}$ et al (2017) Microglial TNF alpha induces COX2 and PGI2 synthase expression in spinal endothelial cells during neuropathic pain. ENeuro. https://doi.org/10.1523/ENEURO.0064-17. 2017

12. Liang F et al (2017) Dexmedetomidine attenuates neuropathic pain in chronic constriction injury by suppressing NR2B, NF- $\mathrm{BB}$, and iNOS activation. Saudi Pharmaceu J 25(4):649-654

13. Takeda $\mathrm{K}$ et al (2005) Role for cyclooxygenase 2 in the development and maintenance of neuropathic pain and spinal glial activation. Anesthesiology 103(4):837-844

14. Shim HS et al (2019) Peripheral and central oxidative stress in chemotherapy-induced neuropathic pain. Mol Pain 15:1744806919840098

15. Shaheen $F$ et al (2010) In vitro assessment of cytotoxicity, antiinflammatory, antifungal properties and crystal structures of metallacyclic palladium (II) complexes. J Organomet Chem 695(3):315-322

16. Shi C-Y et al (2010) Synthesis, crystal structure, DNA-binding and cytotoxicity in vitro of novel cis-Pt (II) and trans-Pd (II) pyridine carboxamide complexes. Bioorg Med Chem Lett 20(24):7250-7254

17. El-Aarag BY et al (2014) In vitro anti-proliferative and antiangiogenic activities of thalidomide dithiocarbamate analogs. Int Immunopharmacol 21(2):283-292

18. Cuzzocrea $S$ et al (2002) Pyrrolidine dithiocarbamate attenuates the development of acute and chronic inflammation. Br J Pharmacol 135(2):496-510

19. Naveed $M$ et al (2019) A new cationic palladium (II) dithiocarbamate exhibits anti-inflammatory, analgesic, and antipyretic activities through inhibition of inflammatory mediators in in vivo models. Naunyn Schmiedebergs Arch Pharmacol 392(8):961-977

20. Tsutsumi K et al (2016) Polaprezinc reduces paclitaxel-induced peripheral neuropathy in rats without affecting anti-tumor activity. J Pharmacol Sci 131(2):146-149

21. Khan $\mathrm{S}$ et al (2013) Mechanism underlying anti-hyperalgesic and anti-allodynic properties of anomalin in both acute and chronic inflammatory pain models in mice through inhibition of NF- $\kappa \mathrm{B}, \mathrm{MAPKs}$ and CREB signaling cascades. Eur J Pharmacol 718(1-3):448-458

22. Khan $\mathrm{S}$ et al (2014) Anti-hyperalgesic and anti-allodynic activities of capillarisin via suppression of inflammatory signaling in animal model. J Ethnopharmacol 152(3):478-486

23. Khan AU et al (2021) Inhibition of NF- $\mathrm{\kappa B}$ signaling and HSP70/ HSP90 proteins by newly synthesized hydrazide derivatives in arthritis model. Naunyn-Schmiedeberg's Arch Pharmacol 394:1-23

24. Khan A et al (2019) Anomalin attenuates LPS-induced acute lungs injury through inhibition of AP-1 signaling. Int Immunopharmacol 73:451-460

25. Ullah MZ et al (2018) Attenuation of inflammatory pain by puerarin in animal model of inflammation through inhibition of proinflammatory mediators. Int Immunopharmacol 61:306-316

26. Zeeshan S et al (2019) N-Pyrazoloyl and N-thiopheneacetyl hydrazone of isatin exhibited potent anti-inflammatory and 
anti-nociceptive properties through suppression of NF-кB, MAPK and oxidative stress signaling in animal models of inflammation. Inflamm Res 68(7):613-632

27. Khalid S et al (2019) Suppression of TRPV1 and P2Y nociceptors by honokiol isolated from Magnolia officinalis in 3rd degree burn mice by inhibiting inflammatory mediators. Biomed Pharm 114:108777

28. Ur Rehman F et al (2021) Surface modified multifaceted nanocarriers for oral non-conventional cancer therapy; synthesis and evaluation. Mater Sci Eng C 123:111940

29. Khan J et al (2019) Attenuation of vincristine-induced neuropathy by synthetic cyclohexenone-functionalized derivative in mice model. Neurol Sci 40(9):1799-1811

30. Dong J et al (2019) Berberine ameliorates diabetic neuropathic pain in a rat model: involvement of oxidative stress, inflammation, and $\mu$-opioid receptors. Naunyn Schmiedebergs Arch Pharmacol 392(9):1141-1149

31. Yemitan OK, Adeyemi OO (2017) Mechanistic assessment of the analgesic, anti-inflammatory and antipyretic actions of Dalbergia saxatilis in animal models. Pharm Biol 55(1):898-905

32. Jung $\mathrm{Y}$ et al (2017) Anti-allodynic effect of Buja in a rat model of oxaliplatin-induced peripheral neuropathy via spinal astrocytes and pro-inflammatory cytokines suppression. BMC Complement Altern Med 17(1):48

33. Ahmad $\mathrm{N}$ et al (2017) A novel pregabalin functionalized salicylaldehyde derivative afforded prospective pain, inflammation, and pyrexia alleviating propensities. Arch Pharm 350(6):e201600365

34. Lee B-H et al (2016) Gintonin enhances performance of mice in rotarod test: involvement of lysophosphatidic acid receptors and catecholamine release. Neurosci Lett 612:256-260

35. Khalid $\mathrm{S}$ et al (2018) Antihyperalgesic properties of honokiol in inflammatory pain models by targeting of NF- $\mathrm{BB}$ and Nrf2 signaling. Front Pharmacol 9:140

36. Khan $\mathrm{S}$ et al (2014) Anti-inflammatory properties of samidin from Seseli resinosum through suppression of NF- $\mathrm{\kappa B}$ and AP-1-mediated-genes in LPS-stimulated RAW 2647 cells. Arch Pharm Res 37(11):1496-1503

37. Khan $\mathrm{S}$ et al (2013) Molecular mechanism of capillarisinmediated inhibition of MyD88/TIRAP inflammatory signaling in in vitro and in vivo experimental models. J Ethnopharmacol 145(2):626-637

38. Khan A et al (2019) Antinociceptive properties of 25-methoxy hispidol A, a triterpinoid isolated from Poncirus trifoliata (Rutaceae) through inhibition of NF-kB signalling in mice. Phytotherapy Res 33(2):327-341

39. Khan A et al (2019) Matrine ameliorates anxiety and depressionlike behaviour by targeting hyperammonemia-induced neuroinflammation and oxidative stress in $\mathrm{CCl} 4$ model of liver injury. Neurotoxicology 72:38-50
40. Khan AM et al (2020) Continentalic acid exhibited nephroprotective activity against the LPS and E coli-induced kidney injury through inhibition of the oxidative stress and inflammation. Int Immunopharm 80:106209

41. Khan S et al (2011) Suppression of LPS-induced inflammatory and NF- $\mathrm{\kappa B}$ responses by anomalin in RAW 2647 macrophages. J Cell Biochem 112(8):2179-2188

42. Shal B et al (2019) Effect of 25-methoxy hispidol A isolated from Poncirus trifoliate against bacteria-induced anxiety and depression by targeting neuroinflammation, oxidative stress and apoptosis in mice. Biomed Pharmacother 111:209-223

43. Khan AU et al (2020) The newly synthesized compounds (NCHDH and NTHDH) attenuates LPS-induced septicemia and multi-organ failure via Nrf2/HO1 and HSP/TRVP1 signaling in mice. Chemico-Biol Interact 329:109220

44. Atiq A et al (2019) Diadzein ameliorates 5-fluorouracil-induced intestinal mucositis by suppressing oxidative stress and inflammatory mediators in rodents. Eur J Pharmacol 843:292-306

45. Kazmi $Z$ et al (2020) Anti-epileptic activity of daidzin in PTZinduced mice model by targeting oxidative stress and BDNF/ VEGF signaling. NeuroToxicol 79:150-163

46. Shal B et al (2020) Neuroprotective effect of 25-Methoxyhispidol A against $\mathrm{CCl}$-induced behavioral alterations by targeting VEGF/ BDNF and caspase-3 in mice. Life Sci 253:117684

47. Ali $\mathrm{H}$ et al (2020) Attenuation of LPS-induced acute lung injury by continentalic acid in rodents through inhibition of inflammatory mediators correlates with increased $\mathrm{Nrf} 2$ protein expression. BMC Pharmacol Toxicol 21(1):1-14

48. Chun J et al (2012) Alantolactone suppresses inducible nitric oxide synthase and cyclooxygenase- 2 expression by down-regulating NF- $\kappa B$, MAPK and AP-1 via the MyD88 signaling pathway in LPS-activated RAW 264.7 cells. Int Immunopharmacol 14(4):375-383

49. Hughes JP et al (2012) Understanding chronic inflammatory and neuropathic pain. Ann N Y Acad Sci 1255(1):30-44

50. Moalem G, Tracey DJ (2006) Immune and inflammatory mechanisms in neuropathic pain. Brain Res Rev 51(2):240-264

51. Marchand F, Perretti M, McMahon SB (2005) Role of the immune system in chronic pain. Nat Rev Neurosci 6(7):521-532

52. Ledeboer A et al (2007) Intrathecal interleukin-10 gene therapy attenuates paclitaxel-induced mechanical allodynia and proinflammatory cytokine expression in dorsal root ganglia in rats. Brain Behav Immun 21(5):686-698

Publisher's Note Springer Nature remains neutral with regard to jurisdictional claims in published maps and institutional affiliations. 